\title{
Progress on the Gemini High-Resolution Optical SpecTrograph (GHOST) design
}

\author{
Michael J. Ireland ${ }^{a}$, Andre Anthony ${ }^{b}$, Greg Burley ${ }^{b}$, Eric Chisholm ${ }^{b}$, Vladimir Churilov ${ }^{c}$, \\ Jennifer Dunn ${ }^{b}$, Gabriella Frost ${ }^{c}$, Jon Lawrence ${ }^{c}$, David Loop ${ }^{b}$, Peter McGregor ${ }^{a}$, \\ Sarah Martell ${ }^{d}$, Alan McConnachie ${ }^{b}$, Richard M. McDermid ${ }^{c, e}$, John Pazder $^{b}$, Vlad Reshetov ${ }^{b}$, \\ J. Gordon Robertson ${ }^{c}$, Andrew Sheinis ${ }^{c}$, Julia Tims ${ }^{c}$, Peter Young ${ }^{a}$ and Ross Zhelem ${ }^{c}$ \\ ${ }^{a}$ Research School of Astronomy and Astrophysics, \\ Australian National University, Canberra ACT, Australia; \\ ${ }^{b}$ NRC-Herzberg Institute of Astrophysics, Victoria, BC, Canada V9E 2E7; \\ ${ }^{c}$ Australian Astronomical Observatory, North Ryde, NSW 2113, Australia; \\ $d$ School of Physics, University of New South Wales, Sydney, NSW 2052, Australia; \\ ${ }^{e}$ Macquarie University, North Ryde, NSW 2109, Australia.
}

\begin{abstract}
The Gemini High-Resolution Optical SpecTrograph (GHOST) is the newest instrument being developed for the Gemini telescopes, in a collaboration between the Australian Astronomical Observatory (AAO), the NRC Herzberg in Canada and the Australian National University (ANU). We describe the process of design optimisation that utilizes the unique strengths of the new partner, NRC - Herzberg, the design and need for the slit viewing camera system, and we describe a simplification for the lenslet-based slit reformatting. Finally, we outline the updated project plan, and describe the unique scientific role this instrument will have in an international context, from exoplanets through to the distant Universe.
\end{abstract}

Keywords: spectrograph, spectroscopy, échelle, high resolution, radial velocity

\section{INTRODUCTION}

The Gemini telescopes are efficient, $8 \mathrm{~m}$ telescopes with a wide range of capabilities and a broad science community. However, the one demanded capability that the telescopes have lacked is a high resolution optical spectrograph. Previous concepts have been proposed, ${ }^{1}$ and have either suffered from flexure and space constraints in one of the instrument ports at Cassegrain, or limit throughput due to losses from fibers. The limitations due to fibers are no longer as relevant as they once were, with greatly improved throughputs, especially at wavelengths longer than $350 \mathrm{~nm}$.

In late 2011, the Gemini telescopes awarded three competitive conceptual design studies for the Gemini High-Resolution Optical SpecTrograph (GHOST) concept. The Australian Astronomical Observatory (AAO) in partnership with the Australian National University (ANU) conducted one of these studies, with a fiber-fed design incorporating a spectrograph designed by the New Zealand company, Kiwistar Optics. Many of the details of this design are already published, ${ }^{2}$ and we aim to minimise the repetition of those details here.

In the process of contractual negotiations, Kiwistar Optics were not able to commit to acting as a subcontractor for this project, and the project team was re-formed with the National Research Council (NRC) Canada's NRC - Herzberg (who conducted another conceptual design study) taking on the role of constructing the spectrograph from the fiber slit to detector. Contracts were signed in April 2014, with a kick-off meeting in May 2014. The AAO is maintaining overall project leadership, and is constructing the fiber and on-telescope components, with the Australian National University (ANU) providing instrument control software. The preliminary design stage for GHOST will finish at the end of 2014.

Further author information: (Send correspondence to M.Ireland.) M.Ireland.: E-mail: michael.ireland@anu.edu.au A.Sheinis.: E-mail: asheinis@aao.gov.au; D. Loop: E-mail: David.Loop@nrc-cnrc.gc.ca

Ground-based and Airborne Instrumentation for Astronomy V, edited by

Suzanne K. Ramsay, lan S. McLean, Hideki Takami, Proc. of SPIE Vol. 9147,

$91471 \mathrm{~J} \cdot$ ? C 2014 SPIE · CCC code: 0277-786X/14/\$18 · doi: 10.1117/12.2057356

Proc. of SPIE Vol. 9147 91471J-1 


\section{SCIENCE CONTEXT}

The primary science context of GHOST was laid out in the 2010 call for white papers from the Gemini observatory. The largest community need for GHOST was clearly as a means to measure elemental abundances of stars, especially metal-poor stars. The measurement of abundances of the oldest stars in our Galaxy, enables us to piece together how the first generation of stars died, ${ }^{3}$ enables us to date the oldest stars directly, ${ }^{4}$ and enables the piecing together of the formation of our Galaxy (so-called "Galactic archaeology" ${ }^{5}$ ). These science cases require the broadest possible wavelength coverage, a spectral resolving power of at least $\mathrm{R}=\lambda / \Delta \lambda \sim 30,000$ (with higher resolutions up to $\mathrm{R} \sim 100,000$ often preferable at least until readout noise dominates) and often have more than one target per 7 arc-minute diameter Gemini field of view.

The science context of Gemini has changed somewhat since 2010. In this period, a large amount of Keck HIRES time was taken up with transiting exoplanet follow-up from the Kepler mission, demonstrating a need for large telescope precision radial velocity observations. This need will not go away: the next decade brings two new transiting exoplanet space missions (TESS and PLATO) which will produce a very large number of candidate exoplanets at a magnitude of $\mathrm{R} \sim 12$, where $1-4 \mathrm{~m}$ telescopes arguably do not provide the most efficient means of follow-up. Extreme precision is not required for much of this follow-up: some follow-up is possible at a precision of $20 \mathrm{~ms}^{-1}$, and at $2 \mathrm{~ms}^{-1}$ GHOST would become a competitive exoplanet follow-up instrument, enabling follow-up of hot Neptunes discovered with TESS and PLATO.

\section{INSTRUMENT OVERVIEW}

Although a comprehensive description of the conceptual design has been given before ${ }^{2}$ there are some changes or potential changes during preliminary design due to the unique strengths of NRC - Herzberg and additional capabilities of vendors for fibers and micro-optics. The key properties of GHOST at this point in preliminary design are:

- Simultaneous wavelength coverage from $363 \mathrm{~nm}$ to at least $900 \mathrm{~nm}$.

- Two moveable Integral Field Units (IFUs): one with a 19-element, 1.2" diameter IFU and a 7-element, 1.2" diameter IFU, and one with a 7-element, 1.2" diameter IFU only (plus sky fibers).

- A high-resolution mode with $\mathrm{R}>75,000$ for the 19-element IFU, and a standard resolution mode with $\mathrm{R}>50,000$ for the 7 -element IFU.

- Guide fibers (instead of an on-instrument wavefront sensor), re-imaged to their own camera.

- A fiber slit viewing and exposure meter camera, extracting approximately $0.5 \%$ of the broad-band light entering the spectrograph.

- Simultaneous Th/Xe lamp calibration for the high resolution mode, and fiber agitation to avoid modal noise.

- An asymmetric white-pupil échelle spectrograph, with resolution equivalent to an R3.7, 100mm design.

\section{IFU IMAGE SLICING CONCEPT}

The integral field unit image-slicing concept follows closely the concept of the KOALA Integral Field Init (IFU) instrument at the Anglo-Australia Telescope, ${ }^{6}$ where a field of view of up to $49 \times 27$ arc sec is sampled by 1000 hexagonal microlenses, with a factor of $\sim 4$ increase in blue $(\sim 390 \mathrm{~nm})$ throughput over its predecessor, SPIRAL. ${ }^{7}$ The key difference between KOALA and GHOST is that while the IFU in KOALA is designed to sample many semi-independent spatial elements, the IFU in GHOST is simply a means of sub-sampling the seeing disk, so that the "slit losses" are small while the effective slit width-resolution product of the spectrograph can also be small, enabling a smaller and more cost-effective spectrograph. The integral field unit format on one of the probes is shown in Figure 1. The slit length needed to accommodate one object plus calibration and sky fibres at high resolution $(\mathrm{R}>75,000)$ can alternatively accommodate two objects (or one object plus independent sky) 

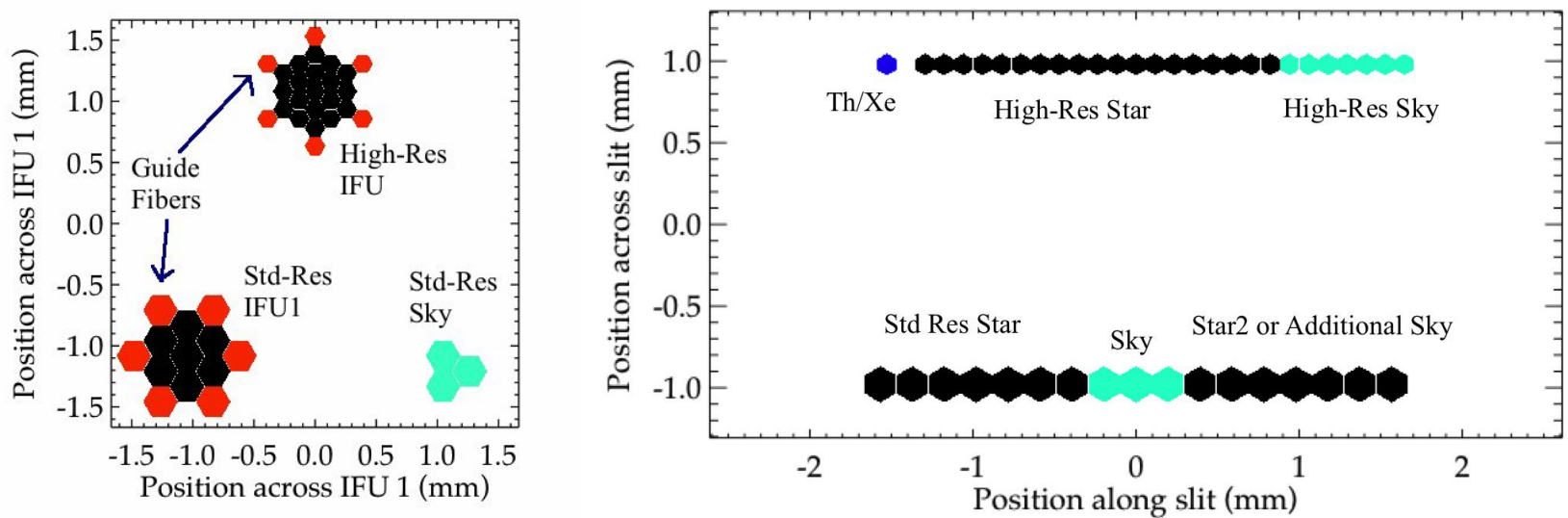

Figure 1. The integral field unit on one of the probes (left) and the format in the slit plane (right). Optimising the probe and slit layout is part of preliminary design.

at standard resolution. As the additional cost and complexity of two moving object IFUs was small compared to the whole project, and also enables the expansion to a polarimetry mode, we decided from the conceptual design proposal stage to have two moving objects.

Apart from a 15-20\% allowance for focal-ratio degradation, the input and output optics have the same format (and, unlike the concept presented in the previous SPIE paper, ${ }^{2}$ it appears likely that both resolution modes will be possible with lenslet arrays only, without bulk optics). This means that the telescope pupil is imaged on to the fiber face, and the fiber face is imaged on to the grating (and the white pupil). Changes in focal-ratio degradation or injection therefore have a very small effect on the spectrograph pupil illumination. An even pupil illumination means that the point-spread function of the spectrograph is expected to be stable to a higher degree than typical of fiber-fed spectrographs. The down-side of this design is that the slit illumination is expected to change with seeing and variable focal-ratio degradation. An example slit illumination is shown in Figure 2. Although the micro-lenses on output are hexagonal, each fiber output has a circular profile, with the edge of the hexagon only visible at high contrast, in the case of extreme misalignment (as in Figure 2) or in the case of unusually large Focal-Ratio Degradation (FRD). The simulation in Figure 2 only modelled FRD by convolution 1 degree full-width-half-maximum angular profile.

The change in slit profile due to moderate changes in seeing and alignment is anticipated to affect the radial velocity at the $2 \mathrm{~ms}^{-1}$ level. This figure is derived from a slit profile centroid when compared to the Th/Xe lamp so is a lower limit - there are additional error terms to be discussed below.

\subsection{Slit Viewing Camera}

Having established that the slit viewing camera is essential for $<2 \mathrm{~ms}^{-1}$ radial velocity accuracy, and given that the instrumental bandpass is much broader than the peak radial velocity sensitivity bandpass for any given spectral type, we are considering a slit viewing camera design that measures the slit profile in two filter bandpasses. The baseline design is shown in Figure 3. Rather than a filter wheel to give colour information (with a loss in photon rate), our preferred design uses dichroic mirrors to split the primary precision radial velocity bandpass into a red and a blue bandpass (Figure 4), with both bandpasses imaged simultaneously on the one camera.

\subsection{Radial Velocity Precision}

The radial velocity precision error budget to be completed during preliminary design contains the following terms for the precision requirement (now proposed by the instrument team as $20 \mathrm{~ms}^{-1}$, with $2 \mathrm{~ms}^{-1}$ as a goal):

- Changing point-spread function due to changes in pupil illumination, differentially affecting the reference and the star slit elements. 

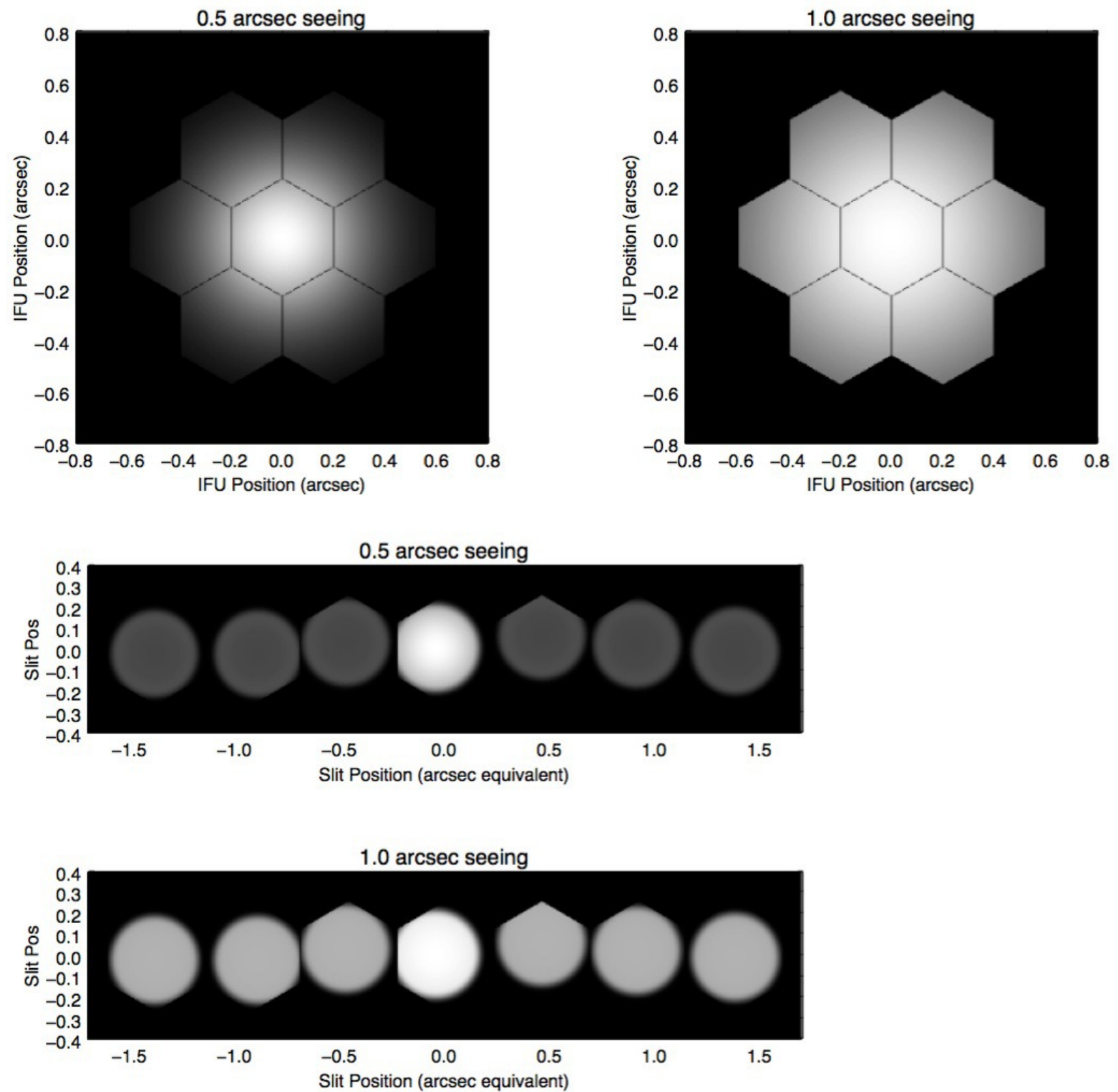

Figure 2. A simulated star image at 0.5 and 1.0 arcsec seeing as seen on a simulated standard resolution integral field unit. The relative decentering of each slit element is due to tilt errors in the fiber array - exaggerated here for visibility by a factor of 2 from an achievable (and prototyped) specification. In this arrangement in standard resolution mode, a change in seeing from 0.5 to 1.0 arcsec produces a calculated radial velocity shift of $12 \mathrm{~ms}^{-1}$, demonstrating the need for slit imaging to achieve precision radial velocity. More realistic fiber tilts for the high-resolution mode results in only a $2 \mathrm{~ms}^{-1}$ radial velocity error. 


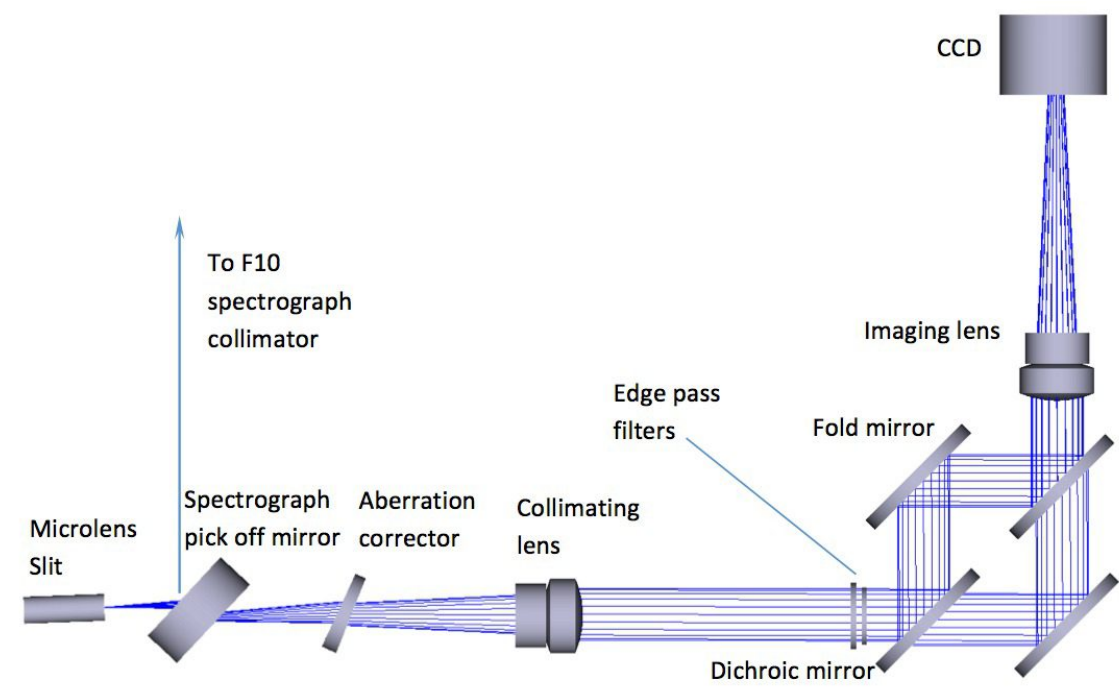

Figure 3. An updated baseline design for the slit viewer (note that in this design, the guide fibers are imaged separately). A leaky dielectric mirror reflects the f/10 beam to the spectrograph, with approximately $0.5 \%$ of the broad-band light being transmitted to the slit viewing optics. The collimating and imaging lenses are both microscope tube lenses, and one of the fold mirrors is tilted so that the blue and red slit images are seen by different parts of the CCD. An off-the-shelf CCD package such as the SX-694 from Starlight Express (several under consideration) will be used with appropriate binning to sample each lenslet with approximately $4-5$ pixels.

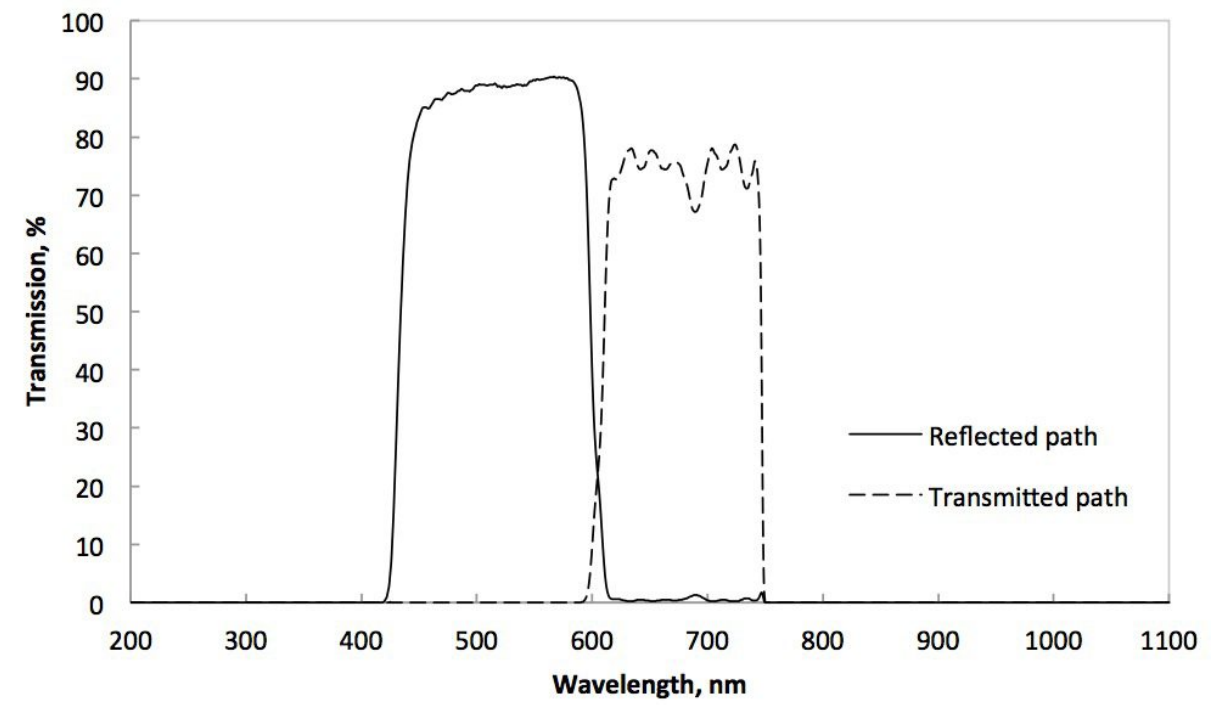

Figure 4. The bandpasses for the red and blue slit images, covering the wavelengths expected to be used for precision radial velocity, and not including spectral regions where sky will most often dominate over the stellar spectrum. 
- Change in slit tilt due to thermal changes in the spectrograph, differentially affecting the reference and the star slit elements.

- Changes in point-spread function due to e.g. thermal changes in the instrument (despite the temperature control).

- Errors in measuring slit illumination and applying the measurement in the software pipeline.

- Drifts in the reference lamp.

To achieve the $2 \mathrm{~ms}^{-1}$ goal, long-term changes in slit tilt or point-spread function are acceptable as long as they can be sensed and taken into account in the analysis pipeline - only the un-sensed changes are important in the error budget.

\section{SOFTWARE AND OPERATIONS CONCEPT}

\subsection{Operational Modes}

GHOST is intended to be a fixed format instrument with four main operational modes, two of which will be offered with at least two types of on-chip binning:

1. Dual-object, standard resolution (no binning or $\sim 4 \times 2$, spatial $\times$ wavelength binning).

2. Object plus sky, standard resolution (no binning or $\sim 4 \times 2$ binning).

3. Object plus sky, high resolution (no binning or $\sim 4 \times 1$ binning).

4. Object plus sky, high resolution with simultaneous reference.

Each of these modes could potentially be used on targets that are too faint or complex for the guide fibers to acquire the science target, but there will always be a suitable target for acquisition within the 7 arc minute field of view of Gemini. This means that the acquisition process can always check pointing/flexure prior to acquiring the science target and after locking the Peripheral Wavefront Sensor. The Peripheral Wavefront Sensor is always required to track low order modes. (e.g. focus).

The temperature stability of the spectrograph combined with a small set of operating modes will enable relatively robust testing of analysis software, which will be written in Python under the Gemini "recipe system". The most challenging part of the analysis software will be taking into account changing slit illumination for the precision radial velocity mode. We are considering implementing an analysis mode where radial velocity is fitted directly to the combination of the pixel data, a reference spectrum and the slit illumination profile.

\subsection{Control Software}

The software control system for GHOST will be based on the same software developed by the ANU for previous Gemini instruments - GSAOI and NIFS. These software solutions are derived directly from the same codebase as the the ANUs configurable control and data acquisition system - CICADA - with appropriate modifications/enhancements added to support the GHOST functional requirements. The chief advantage of adopting this solution is the benefit of code maturity with the expected commensurate gains in code development time.

Where the GHOST software will differ from GSAOI and NIFS is in the use of the new Gemini instrument software framework - the Gemini Instrument Application Program Interface (GIAPI) - which was first used for the Gemini Planet Imager (GPI). Our intended approach to using GIAPI will be similar to the approach that was used for GSAOI and NIFS with EPICS - a thin-layer approach. This was very successful and allowed a deep re-use if CICADA code with a layer of interface code put in place containing all the EPICS communication specifics. For GIAPI a new communication layer will be written for handling status, alarms, logging/messaging, commands and monitors. This module will be prototyped during the preliminary design. 


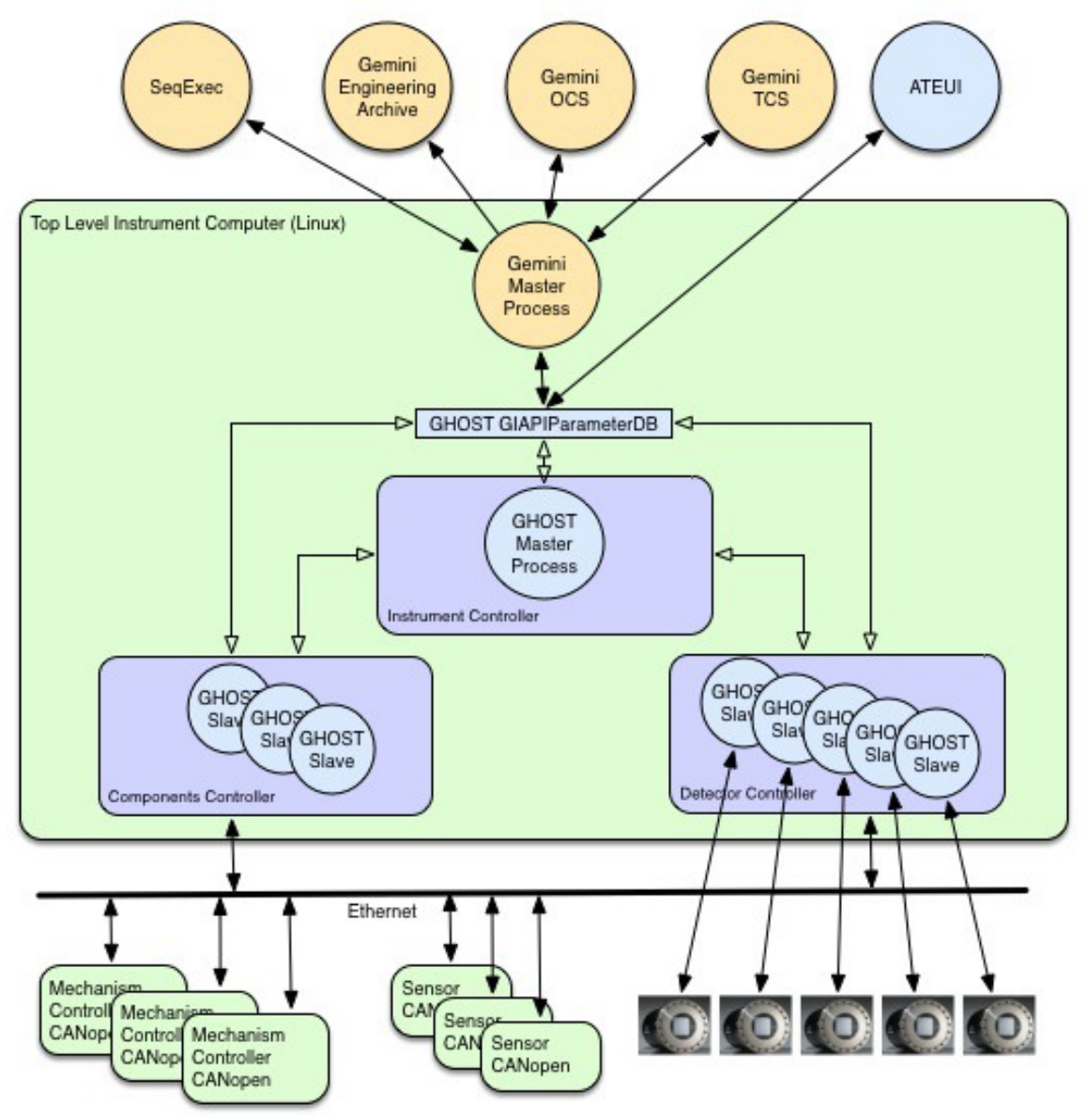

Figure 5. The control software architecture for GHOST.

As mentioned earlier, CICADA is a configurable system and is deployed in a distributed way according to the set of components required for operating the instrument. For GHOST this will include a number of cameras - up to four science, one each for the spectrograph arms (number to be determined during the preliminary design), and two field viewing/guide cameras. In addition there are components for each of the mechanisms and sensors for both the spectrograph and cassegrain sub-systems.

The architecture for the GHOST configuration is shown in Figure 5. Each 'Slave' component in this architecture attends to a single component (or group of related components) of the configuration. CAN bus will be used as the mechanism control system for GHOST and there will be a separate CAN bus instance for each of the two sub-systems - each instance attended to by a 'Slave' component that will address each individual mechanism or sensor. This architecture will allow simultaneous monitoring and control of all cameras, mechanisms and sensors in the system. This is all orchestrated by the 'Master' process.

For GHOST a number of modifications/enhancements to CICADA are required:

- The GIAPI interface module - in the form of a CICADA parameter database.

- Commands are delivered to CICADA by way of messages - with each component typically able to handle one command at a time. With GIAPI, command listeners will be added that intercept commands from the Gemini Observatory Control System or engineering GUI and pass them by way of a message to the 'Master processs command queue. 
- A completely new engineering GUI will be built specifically for the instrument and probably with Java (including JavaFX) components to fit in with current Gemini strategy and allow platform independent operation (with hopefully IOS operation with technologies such as RoboVM).

- Support for Geminis notion of system health - based on a hierarchy of health states.

- An alarms system built into CICADAs parameter database.

- Slave components that can handle CAN bus mechanisms.

- Camera interface modules if needed (depends on the camera hardware selected) - CICADA already supports a range of camera controllers including those from Astronomical Research Cameras - a candidate under consideration.

- Improvements to CICADAs command parsing and parameters definition modules

\section{PRELIMINARY DESIGN TRADES}

The original GHOST concept involved a modied Kiwispec design (a product of Kiwistar Optics), which was a 3:1 asymmetric white-pupil échelle. The limited bandpass of the Volume Phase Holographic (VPH) cross-dispersers in this design was the primary driver in the conceptual design to a 4-arm design. NRC-Herzberg is leading a trade study of several designs. This design study is leveraging the recent progress in VPHG production and CCD sensitivities. Slant grove VHG's are now regularly produced. Considerable progress has also been made on the production of low frequency VPGH's with line frequencies below $500 \mathrm{G} / \mathrm{mm}$. Low line frequency grating are required for the red grating and the limited ability to produce gratings with low enough line frequencies have required the used of a white pupil asymmetry to bring the line frequency within the manufacturable range. The E2V Deep depletion CCD with the DD astro multi-2 coating has a QE at 400nm of $90 \%$ without reduced the $\mathrm{QE}$ in the red when compared to the previous E2V coatings. This coating gives the performance of a Graded coating without the risk. The goal of the design study is to improve the system efficiency, especially in the blue. The design options being considered are:

- Consideration of an R2 grating, reducing beam anamorphism, providing a more uniform dispertion across the orders.

- Optimization of the pupil asymmetry of the white pupil design in conjunction with the optimization of the VPHG parameters to improve the broad-band and blue efficiency, possibility reducing the number of arms to 3 , or even 2 .

- Incorporation of the most recent detector developments, including the recent progress on $\mathrm{QE}$ and the availability larger detectors. dielectric broad band coatings on the white pupil relay, potentially improving the mirror reflectivity to $>98 \% \mathrm{R}$ over the band, and tuning the coating design for $>99 \% \mathrm{R}$ in the blue.

The optical layout will remain as a white pupil design because of the stray light and radial velocity performance advantages. An example design being considered is shown in Figure 6.

\subsection{Performance Metrics}

In optimising the GHOST design during preliminary design, we will be trading performance against cost, schedule and risk. This trade includes both spectrograph optimisations and deciding on the precise spectrograph location (which affects the fiber length and throughput). Rather than fixing all conceptual design requirements as absolute, we are considering designs which exceed and in some cases to not meet conceptual design requirements as a trade against cost, schedule and risk.

The easiest to understand performance figure is observational efficiency: if this decreases by $10 \%$, then $10 \%$ more observing time is required over the lifetime of the instrument, and naively, this is equivalent to a cost of $10 \%$ of the lifetime value of the telescope time anticipated for GHOST. This lifetime value is $\sim 600$ nights 


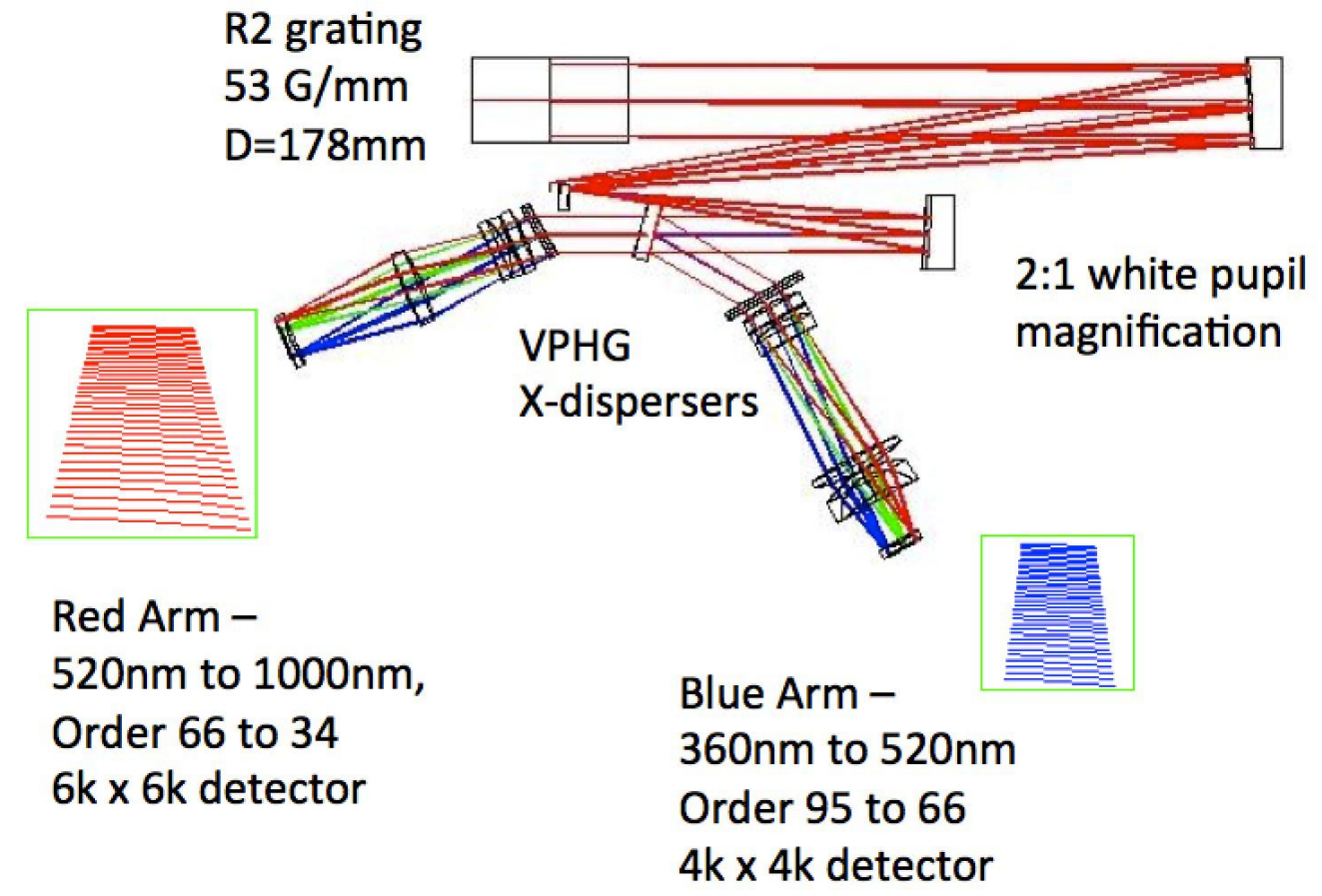

Figure 6. One example (out of several) designs being considered by NRC-Herzberg in the early part of the preliminary design optimisation.

with a value of $\sim \$ 65,000$ per night (scaling reported values of other telescopes by area), giving $\sim \$ 4 \mathrm{M}$ for this hypothetical $10 \%$ hit. Of course, the marginal utility of telescope time is less than its lifetime averaged utility, as the most productive science is ranked most highly and will completed even if the efficiency is low. Given the complexity of the performance versus cost (plus risk and schedule) trades, the final decision on the preferred key preliminary design trades will be made by Gemini, after input from the science and technical teams on the design options. The key performance metrics are:

- Observational efficiency. e.g. The removal of the need to take regular calibration exposures during dark time increases the value of the instrument. Science cases with long exposures and many shorter ( 15 minutes) exposure sequences are both taken into account.

- Throughput, weighted towards the blue (375-400 nm wavelengths in particular). For most of the GHOST science cases, a 10\% throughput gain over the baseline would equate to a 10\% improvement in observational efficiency.

- Low SNR performance. Although much of the GHOST science requires high signal-to-noise (e.g. small equivalent width lines) and is therefore photon-limited, some science will inevitably be done at low SNR. For these science cases, readout noise, number of pixels read out, number of sky IFU elements and possibly dark current are important. After applying a multiplier for the fraction of low SNR science, it is relatively easy to compute an effective loss in observational efficiency due to low SNR performance.

- Minimum and Average Resolution (High Resolution mode). Image quality and pixel sampling affects the spectral resolution in the high resolution mode. For the fraction of science that needs this mode, we will approximate that a $10 \%$ resolution gain would be equivalent to a $10 \%$ gain in observational efficiency (justified from photon-limited signal-to-noise arguments for equivalent width measurements of isolated lines). 
- Long-wavelength science. If the performance at wavelengths longer than $900 \mathrm{~nm}$ is decreased below the baseline (e.g. by not sampling the full free spectral range of the longest wavelength orders), an appropriate penalty will be applied to the instrument performance metric.

- Spectral Stability. Point spread function shape and stability, although difficult to accurately compare between designs, affects any precision radial velocity mode. During preliminary design, we will attempt to convert these design parameters into a radial velocity precision gain or loss, with a correspondingly increased or decreased science reach (similar to the long-wavelength science).

\section{CONCLUSIONS}

The Gemini High-resolution Optical SpecTrograph (GHOST) has now begun preliminary design, with an instrument team lead by AAO and including NRC-Herzberg and ANU. The design includes the full 363nm to $1000 \mathrm{~nm}$ spectral range at fixed format, with either one object at $\mathrm{R}>75,000$ including a simultaneous reference or two objects at $\mathrm{R}>50,000$. One goal of the high-resolution mode is to produce radial velocities to better than $2 \mathrm{~ms}^{-1}$ : we anticipate that this will be challenging and is one driver for the requirement of a slit viewing camera, likely with 2 simultaneous filter bandpasses. Trade studies during preliminary design will choose the final spectrograph format, with the most important performance metric being blue throughput. We anticipate the preliminary design review in December 2014, followed by an aggressive schedule. Critical design review is anticipated for mid-2015, with instrument commissioning in mid-2017.

\section{REFERENCES}

1. F. Diego, D. Brooks, A. Charalambous, I. A. Crawford, P. D'Arrigo, M. Dryburgh, H. Jamshidi, A. S. Radley, T. Savidge, and D. D. Walker, "Conceptual design of the high-resolution optical spectrograph for the Gemini South Telescope," in Optical Telescopes of Today and Tomorrow, A. L. Ardeberg, ed., Proc. SPIE 2871, pp. 1126-1134, Mar. 1997.

2. M. J. Ireland, S. Barnes, D. Cochrane, M. Colless, P. Connor, A. Horton, S. Gibson, J. Lawrence, S. Martell, P. McGregor, T. Nicolle, K. Nield, D. Orr, J. G. Robertson, S. Ryder, A. Sheinis, G. Smith, N. Staszak, J. Tims, P. Xavier, P. Young, and J. Zheng, "The AAO's Gemini High-Resolution Optical SpecTrograph (GHOST) concept," in Proc. SPIE 8446, 29, Sept. 2012.

3. S. C. Keller, M. S. Bessell, A. Frebel, A. R. Casey, M. Asplund, H. R. Jacobson, K. Lind, J. E. Norris, D. Yong, A. Heger, Z. Magic, G. S. da Costa, B. P. Schmidt, and P. Tisserand, "A single low-energy, ironpoor supernova as the source of metals in the star SMSS J031300.36-670839.3," Nature 506, pp. 463-466, Feb. 2014.

4. A. Frebel, N. Christlieb, J. E. Norris, C. Thom, T. C. Beers, and J. Rhee, "Discovery of HE 1523-0901, a Strongly r-Process-enhanced Metal-poor Star with Detected Uranium," ApJ 660, pp. L117-L120, May 2007.

5. D. B. Zucker, G. de Silva, K. Freeman, J. Bland-Hawthorn, and Hermes Team, "The Galactic Archaeology with HERMES Survey," in Galactic Archaeology: Near-Field Cosmology and the Formation of the Milky Way, W. Aoki, M. Ishigaki, T. Suda, T. Tsujimoto, and N. Arimoto, eds., Astronomical Society of the Pacific Conference Series 458, p. 421, Aug. 2012.

6. R. Zhelem et. al, "KOALA, a wide-field 1000 element integral-field unit for the Anglo- Australian Telescope: assembly and commissioning," in Proc. SPIE 9147, 134, 2014.

7. M. A. Kenworthy, I. R. Parry, and K. Taylor, "SPIRAL Phase A: A Prototype Integral Field Spectrograph for the Anglo-AustralianTelescope," PASP 113, pp. 215-226, Feb. 2001. 